\title{
Development of selected reaction monitoring-MS methodology to measure peptide biomarkers in prostate cancer
}

\author{
Anastasia K. Yocum ${ }^{1,2,3}$, Amjad P. Khan ${ }^{1}$, Rong Zhao ${ }^{1}$ and Arul M. Chinnaiyan ${ }^{1,2,4,5}$ \\ ${ }^{1}$ Michigan Center for Translational Pathology, Ann Arbor, MI, USA \\ 2 Department of Pathology, University of Michigan, Ann Arbor, MI, USA \\ ${ }^{3}$ Department of Urology, University of Michigan, Ann Arbor, MI, USA \\ ${ }^{4}$ Howard Hughes Medical Institute, University of Michigan Medical School, Ann Arbor, MI, USA \\ ${ }^{5}$ Comprehensive Cancer Center, University of Michigan Medical School, Ann Arbor, MI, USA
}

Prostate cancer is a leading cause of cancer-related death. The current modality of diagnosis, the measurement of serum PSA, not only suffers from lack of specificity, but does not distinguish clinical cases in which current treatment measures would be most successful, i.e. aggressive, life-threatening tumors. A multiplexed MS methodology, selected reaction monitoring-MS/MS coupled with stable isotope dilution (SID), was developed and tested in both cells lines and clinical tissue samples. Standard curves were generated for two peptides representing PSA and one peptide from each of two additional orthogonally validated biomarkers, AMACR and EZH2. The standard curves show high reproducibility, sensitivity, and good linearity. All four peptides were then measured in six clinically relevant cell lines and are in agreement with the biochemical characteristics of each individual cell line. The SID selected reaction monitoring-MS/MS methodology was then transferred to tissue samples, in which the assay shows potential to differentiate benign disease from localized cancer and localized cancer from aggressive metastatic disease. These results establish the preliminary development of a rational targeted MS platform that strives to bridge the gap between discovery and validation of biomarkers for the detection of prostate cancer.

\section{Keywords:}

AMACR / Biomedicine / EZH2 / Prostate-specific antigen / Selected reaction monitoring / Stable isotope dilution

\section{Introduction}

Current screening and diagnosis of asymptomatic prostate cancer (PCA) is by digital rectal examination (DRE) in combination with the serum measurement of prostatespecific antigen (PSA). While this combination of assays is wrought with controversy, it still remains standard mainstay

Correspondence: Dr. Arul M. Chinnaiyan, Investigator, Howard Hughes Medical Institute, Department of Pathology and Urology, University of Michigan Medical School, 1400 E. Medical Center Drive, 5316 UMCCC, Ann Arbor, MI 48109, USA

E-mail: arul@umich.edu

Fax: +1-734-615-4498
Received: January 12, 2010

Revised: May 11, 2010

Accepted: July 15, 2010 for diagnosis of PCA. One of the major concerns regarding the use of PSA is the unusually high rate of over-diagnosed cancers, approximately $29 \%$ of Caucasian and $44 \%$ of African American [1]. Worse, approximately $15 \%$ of recent cohort participants were subsequently diagnosed with PCA despite having below acceptable PSA threshold levels for further diagnostic testing [2]. Hence, utilizing PSA as a

Abbreviations: AMACR, $\alpha$-methylacyl-CoA racemase; DRE, digital rectal examination; EIC, extracted ion chromatogram; EZH2, enhancer of zeste homolog 2; GAPDH, Glyceraldehyde 3phosphate dehydrogenase; GPM, Global Proteome Machine; PCA, prostate cancer; PSA, prostate-specific antigen; SID, stable isotope dilution; SRM, selected reaction monitoring 
diagnostic biomarker for PCA is in question. Now, the goal of early detection in PCA is to find those tumors at a point at which a cure is possible [3]. This requires biomarkers that are not just reflective of the presence of cancer, but more, tell the aggressive nature and possibly the lethality of the disease.

MS-based technologies have been applied to many discovery-based, large-scale studies and mostly applied to the discovery of protein biomarkers, especially in the field of cancer. Collectively these studies have produced thousands of putative biomarkers including several specifically for PCA [4]. Unfortunately, only a few are currently being validated and applied in clinical diagnostics [5, 6] and none have been validated in PCA specifically. A targeted methodology in which to commence biomarker validation is being advanced within the proteomics community [5, 7-9]. It is hypothesized here that selected reaction monitoring MS/MS (SRMMS), when coupled with the introduction of stable isotopically labeled internal standards, can be developed for the verification of putative biomarkers in PCA.

This multiplexing capability of SRM-MS has the potential to improve diagnostic power of PCA. By combining multiple known biomarkers as representatives for different aspects of the disease, a new multiplexed assay could distinguish indolent versus aggressive disease thereby targeting diagnoses where treatment would be most successful. This work describes the development of a new multiplexed assay utilizing stable isotope dilution (SID) SRM-MS. Its intent is to provide the initial step for the development, optimization, and testing proof of principle both technically and biologically before expanding the number of markers and transitioning the assay to a non-invasive biological matrix, postDRE urine. Included in the assay are peptides representative of two proteins, $\alpha$-methylacyl-CoA racemase (AMACR) and enhancer of zeste homolog 2 (EZH2), which were previously characterized as possible PCA biomarkers [10-14].

AMACR and EZH2 proteins have diverse activity and functionality but both have been shown to be involved with PCA in vivo and in vitro. AMACR is an enzyme involved in the conversion of $R$ - to $S$-stereoisomers and peroximsomal $\beta$-oxidation of branched-chain fatty acids. This enzyme has been shown repeatedly to have significant over-expression in both the transcript and protein levels in PCA samples [11, 12]. EZH2 participates in the methylation of lysine 27 in histone $\mathrm{H} 3$, which consequently leads to the repression of many target genes [10]. It has been shown that EZH2 is overexpressed in metastatic cancer and is a marker of aggressive disease in clinically localized prostate tumors [14]. Therefore, both AMACR and EZH2 were chosen as biomarkers of aggressive disease in localized cancer and its malignant potential. Finally, we added two peptides representative of PSA to our panel. This was not only for proof of concept but also in an attempt to correlate the development of this assay to previously obtained clinical PSA ELISA values.

Once developed and optimized analytically for selectivity and sensitivity, the assay was used to demonstrate proof of concept measuring the approximate concentration of each peptide in six immortalized benign and cancerous prostate cell lines. After proof of principle and analytical feasibility was confirmed with these cells lines, the assay was transferred to prostate tissue samples. A total of 15 tissue samples were utilized representing three clinical categories, five benign (adjacent to tumor), five localized cancer (PCA), and five metastatic tumor samples. Pooled samples for each category were also measured by Western blot and corroborate the SIDSRM-MS results. These four peptide markers measured with SID-SRM-MS show potential as a panel to discriminate between localized cancer and metastatic disease as well as differentiating benign adjacent tissues from localized tumor. While preliminary results are shown, we believe this is a novel and necessary step for verification of both the multiplexed SIDSRM-MS technology platform and of these specific peptide biomarkers for the eventual transition of the assay to post-DRE first void urine. It is hoped that additional markers can be included, which will further help in distinguishing indolent versus aggressive PCA, which will ultimately tailor treatment options to those most in need.

\section{Materials and methods}

\subsection{Cell line and tissue sample preparation}

Collection of whole cellular protein extract from cell lines (RWPE, PC3 DU145, 22Rv1, LnCaP, VCaP, and cancer prostate tissue biopsy samples was done in RIPA complete buffer (Pierce Biotechnology) supplemented with Complete Protease Inhibitor Cocktail (Roche). Total protein extract was quantified with bicinchoninic acid (Pierce Biotechnology). Aliquots of $50 \mu \mathrm{g}$ of whole cellular proteins were separated by 1-D SDS-PAGE (4-12\% Bis-Tris Novex-Invitrogen, Carlsbad, CA). Twenty-four equal sized gel bands were excised and subjected to in-gel digestion. Extracted and lyophilized peptides were reconstituted with $12 \mu \mathrm{L}$ of $2.5 \mathrm{fmol} / \mu \mathrm{L}$ ( $30 \mathrm{fmol}$ ) of each isotopically labeled peptide standard prior to a $10 \mu \mathrm{L}$ ( $25 \mathrm{fmol}$ of each internal standard on column) injection on-line reverse phase LC-SRM-MS (1200 Series HPLCCHIP, 6410 Triple Quadrapole, Agilent Technologies). Peptides were eluted on-line to the MS-QQQ with a reverse phase linear gradient from $97 \%$ A $(0.1 \%$ Formic acid in water) to $45 \%$ B $(0.1 \%$ formic acid in acetonitrile). Western blotting was completed in parallel with the following primary antibodies; anti-mouse-EZH2 (BD Biosciences - 612666), anti-mouse-AMACR (Zymed 39-4000), and anti-rabbit-PSA (Zymed 08-0044). Goat anti-HRP IgG mouse and rabbit were used for secondary development (Santa Cruz Biotechnology).

\subsection{Clinical samples}

Benign adjacent prostate and PCA tissues were obtained from a radical prostatectomy series at the University of 
Michigan Hospitals while the metastatic PCA tissues were obtained from the Rapid Autopsy Program, which are both part of the University of Michigan Prostate Cancer Specialized Program of Research Excellence (S.P.O.R.E) Tissue Core. Samples were collected with informed consent and prior institutional review board approval at the University of Michigan. Additional detailed clinical information regarding these tissue samples is provided in Supporting Information. All samples were stripped of identifiers prior to assessment and stored at $-80^{\circ} \mathrm{C}$ until use.

\subsection{Method development}

One 'signature' peptide from each of the putative protein biomarkers, AMACR and EZH2 and an additional two peptides from PSA were chosen to develop a multiplexed SRM-MS assay. Detailed rational for method development is found in Supporting Information, which briefly includes in silicos prediction, spectra database repository and homology search, along with ion trap analyses. The peptide from EZH2 and one peptide from PSA were synthesized with stably isotopically labeled C-terminal lysine resulting in a mass difference of $8 \mathrm{Da}$. The other PSA peptide was synthesized with stably isotopically labeled C-terminal arginine resulting in a mass difference of $10 \mathrm{Da}$. Finally, the peptide AMACR was synthesized with an internal proline residue stably isotopically labeled, resulting in a mass difference of $6 \mathrm{Da}$. Three transitions for each stably isotopically labeled peptide were developed empirically optimizing for both sensitivity and selectivity, data shown in Supporting Information. Dwell time was constant at $45 \mathrm{msec}$ for each transition and unit resolution was utilized for both Q1 and Q3. Standard curves with spiked internal standard were developed for each of the four peptides in a background of whole cell lysate prepared as stated above and are shown in Fig. 1A and in Supporting Information.

The standard curve was generated as followed. Briefly, after in-gel digestion, extracted peptides from the 24 gel segments were re-solubilzed with serial dilutions of each internal standard so that the following concentrations of internal standard were loaded on to the column: 0 amol, 250 amol, 500 amol, $1 \mathrm{fmol}, 5 \mathrm{fmol}, 10 \mathrm{fmol}$, and $50 \mathrm{fmol}$. Three technical replicates of all 24 gel segments, i.e. entire gel-lanes, were analyzed for concentrations $\leq 1 \mathrm{fmol}$ and five technical replicates were completed for concentrations $\geq 5 \mathrm{fmol}$. A five point concentration standard curve was then generated for each peptide. Individual replicate measurement of AUCs are summed across the 24 fractions and transformed into logarithmic scale for ease of interpretation before being plotted. A linear trend line was then drawn for the average $\log _{(2)}$ value and a correlation coefficient calculated. All raw data and plots without logarithmic transformation are included in Supporting Information.

\subsection{Data analysis}

Ion chromatograms for each transition were extracted with a symmetric expansion of $m / z \pm 0.1$. Each extracted ion chromatogram (EIC) was then smoothed with a Gaussian function of 15 points width. Peak areas for each transition were calculated utilizing default MS/MS integration. Peak areas were filtered to remove any peaks $<10 \%$ of largest peak and a signal-to-noise ratio (height:peak-to-peak) of 5 . Each transition passing the above filters and inside a retention time drift of $10 \%$ was manually verified. The filtered peak areas were then summed over each experiment (24 segments), and a ratio, standard deviation, coefficient of variation, and 95\% confidence level with upper and lower limits for each heavy:light transition pair is calculated. While each transition was summed for every gel lane, i.e. all 24 segments, appreciable signals were only found in one to three gel segments in and surrounding the region of expected molecular weight for the protein.

\section{Results and discussion}

Current diagnostic and prognostic tests for PCA have low specificity. This work describes the development of a multiplexed MS methodology for PCA that strives to be more specific, i.e. distinguishing indolent from aggressive disease. Peptides targeted for this assay were chosen from three different proteins, AMACR, EZH2, and PSA. We believe that the combination of these three proteins offer a newer, more rational manner in which to characterize PCA and a starting point to develop a more targeted methodology to detect life-threatening disease, that is, the goal of not just detecting the presence of indolent disease, but to detect the aggressive nature of the disease. AMACR could mark both the presence of an aggressive localized tumor and possibly the transition from a localized to more metastatic disease. Additionally, EZH2 appears to be a biomarker for an aggressive form of PCA. Finally, PSA was included in these studies as a proof of concept. PSA is well characterized in both the cells lines and metastatic clinical samples used in this work. Further, we know that PSA levels decrease upon current PCA treatments.

\subsection{Method development}

Method development consisted of both in silicos and empirical means iteratively, each guiding the other until the final peptide analytes were chosen and optimized for selectivity and sensitivity. Each of the proteins were theoretically digested and fragmented to predict both precursor and product ions. Predicted peptides were then compared with the listing of observed peptides in the Global Proteome Machine (GPM - http://www.thegpm.org/) database. Several peptides were found in the GPM database for PSA 
A
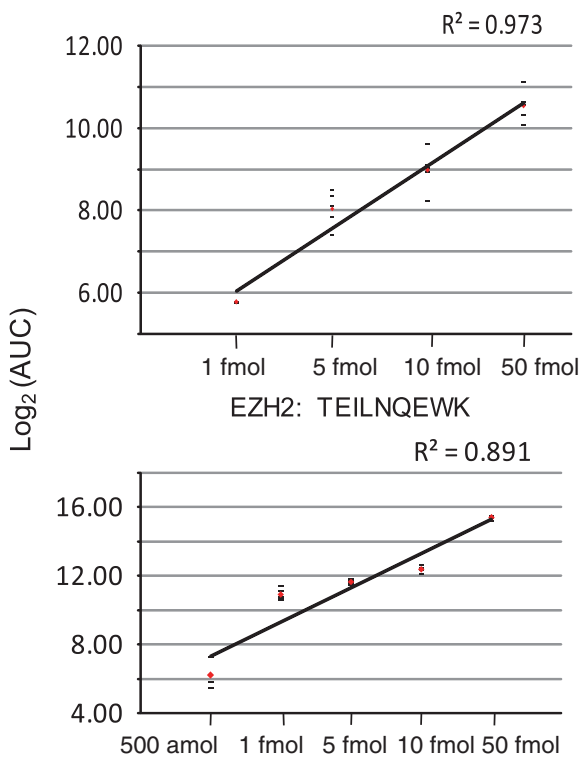

PSA: LSEPAELTDAVK

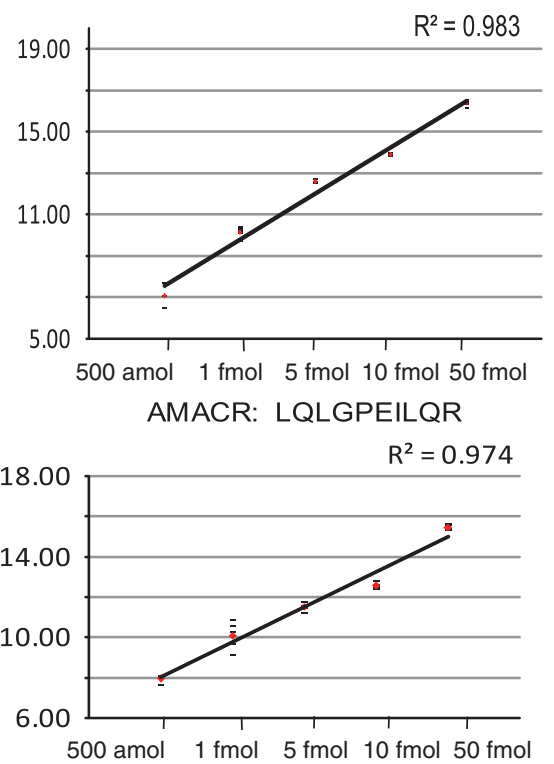

B

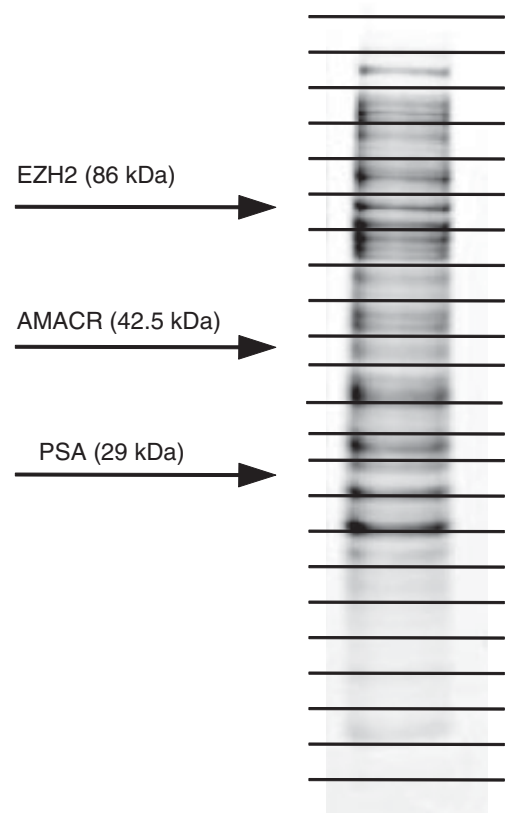

Figure 1. (A) Standard curve for each peptide. For each internal standard, a five concentration $(0.5,1,5,10$, and $50 \mathrm{fmol})$ standard curve was developed over a background matrix of $\mathrm{VCaP}$ whole cellular lysate. Three technical replicates for 500 amol, and five technical replicates for the additional four concentrations are plotted against the $\log _{2}$ translated integrated areas under the curve (AUCs are summed over the 24 fractions) and are represented as black dashes. Averages of those measurements are shown as red diamonds. A linear regression trend line is drawn with indicated $R^{2}$ values. (B) Schematic representation for migration of proteins in 1-D SDS-PAGE. Peptide biomarkers were reproducibly measured by this SID-SRM-MS methodology in 1-D SDS PAGE fractions corresponding at and near the respective protein's molecular weight.

and EZH2; however, none were found for AMACR. Because there were no entries for AMACR in the GPM and the unknown influence of in-gel digestion and peptide extraction, recombinant proteins were analyzed by gel-LC MS/MS on an ion trap. The Method Development Appendix contains the detailed methodologies and tabulated results of both experimental and in silico findings. This iterative process revealed a rationale for culling possible peptide candidates, either predicted or observed, from further method development in the following manner:

(a) Peptides with precursor ions predicted to have a mass outside the mass range of the QQQ were removed.

(b) Peptides that were predicted or observed with a missed cleavage site, a cysteine, methionine, or N-terminal glutamine or glutamate were removed due to the possible variability of experimental modification and artifacts.

(c) Peptides observed experimentally and in the GPM database were then searched in protein BLAST for homology.

This iterative culling revealed a listing of two peptides for EZH2 and three peptides for AMACR to be reasonable candidates for SRM development. Finally, manual inspec- tion of the ion trap spectra resulted in a peptide for each protein that optimized for sensitivity in both precursor and product ion spectra, shown in Supporting Information. These peptides were then transferred to the QQQ utilizing synthesized isotopically labeled heavy amino acid. Peptides for PSA were initially chosen directly from the literature [7, 15]; and, ion trap analysis of the PSA protein confirmed the successful digestion and extraction of the published peptide sequences. Not surprisingly, both peptides utilized for PSA were indeed in the GPM database, however; the final peptide for EZH2 was not listed, nor were any peptides listed for AMACR. This suggests that while looking at experimentally collected data repositories for initial method development may be an advantageous time saver, one may not always obtain the best peptide for SRM method development.

Then, method development commenced on the QQQ for each heavy peptide empirically. Both fragmentor (voltage applied to the exit end of the capillary) and collision energy were empirically optimized for greatest response, see Supporting Information. Each corresponding non-isotopically labeled transition was then added to the instrument method for optimization of dwell time, ensuring for good peak shape, a minimum of 15 points, and a maximum of 25 points across the curve. 
Because the chosen marker proteins, AMACR and EZH2, are expected to be expressed almost ubiquitously in all suitable, analytically comparable, and similar matrixes, empirical method development was completed only on the synthesized heavy peptides. This is in contrast to the method development described by Addona et al., [7], whereby the recombinant light protein and the heavy labeled internal standard peptide are both spiked into the matrix, devoid of endogenous analyte. We feel this approach was not practical in our study as each peptide marker utilized is expected to be detected as a normal endogenous analyte. It is the relative, differential expression of these markers that are biologically significant.

Finally, standard curves for each peptide were generated measuring the heavy peptides in a background of in-gel separated and digested whole cellular lysate matrix to determine approximate sensitivity, selectivity, and linear range of response for each transition. Unfortunately, one peptide from PSA initially chosen for empirical method development did not respond appropriately for sensitivity and was removed from further method development. Shown in Fig. 1A are the summarized results of three replicate measurements for 500 attattmole and five replicate measurements for the remaining increasing concentrations. The approximate LOD of the peptides representing EZH2, AMACR, and one peptide in PSA appears to be approximately 500 attmol and $1 \mathrm{fmol}$ for the other peptide in PSA. The matrix was also analyzed without the addition of any internal standards ( 0 attmol) for which no signal for the internal standards was observed. While not absolute, we believe this to be indicative of good selectivity for the chosen transitions. The calculation of CV in both linear and logarithmic space is shown in the Supporting Information. For both EZH2 and AMACR, at least one transition at 500 attmol had a calculated CV of $\leq 20 \%$, while both peptides in PSA had at least one transition with a calculated $\mathrm{CV} \leq 20 \%$ at $1 \mathrm{fmol}$. Of important note, gel fractionation of whole cellular proteins was found to be necessary for achieving sensitive and selective detection of each peptide in the complex matrix, data not shown. Furthermore, the percent yield of recovering each peptide from in-gel fractionation and tryptic digestion was not determined and calculated. Therefore, this work describes an approximation of quantification, not absolute quantification. This is ongoing and future necessary work.

\subsection{Cell lines}

Having completed the analytical method development, proof of principle was completed for the peptide biomarkers in cell lines. There was initial concern measuring one peptide for each of the EZH2 and AMACR protein given the valid argument by Duncan et al. [9]. Shown in Fig. 1B is a schematic representation of where, with regard to migration in the 1-D SDS-PAGE gel, each peptide was repeatedly measured in all cell lines and tissue samples. The observation that each peptide was measured in the molecular weight region of the respective protein provides confidence that these peptides are good representatives of the protein. However, throughout this text, the term peptide biomarkers will be utilized since this method clearly does not measure the entire protein moiety.

Figure 2 shows EIC for each of the peptides measured. In red are EICs for the spiked internal standard ( $25 \mathrm{fmol}$ ) and in black are the endogenous measurements corresponding to the non-isotopically labeled peptides. All six transitions overlap in retention time and are in agreement in relative ratio to one another. Utilizing fewer transitions per peptide would negate or decrease this confidence $[16,17]$.

Six immortalized cells lines were then used to provide proof of principle for the methodology. Three biological replicate experiments for each cell line were completed. RWPE cells are histologically normal adult human prostate epithelial cells and as such were used as a pseudo negative control for the four peptide biomarkers measured. The data showed negligible or no detection for each of the peptide biomarkers measured; approximately $3.79 \mathrm{fmol}$ for EZH2 and $500 \mathrm{attmol}$ per $50 \mu \mathrm{g}$ of whole cellular lysate for AMACR while non-detectable for both PSA peptides. PC3 cells are prostate adenocarcinoma cells and showed a similar trend; an average of $6.83 \mathrm{fmol}$ for EZH2 and $3.76 \mathrm{fmol}$ for AMACR but again non-detectable for both PSA peptides. Similar results were obtained for DU145 cells, a PSA negative, epithelial prostate carcinoma cells. Average concentration per $50 \mu \mathrm{g}$ of whole cellular lysate was $4.54 \mathrm{fmol}$ for EZH2 and $5.50 \mathrm{fmol}$ for AMACR while non-detectable for both PSA peptides as expected. Interestingly, in 22Rv1 cells, a higher concentration was measured for all four peptide biomarkers, $14.07 \mathrm{fmol}$ for EZH2, $15.86 \mathrm{fmol}$ for AMACR, and $10.82 \mathrm{fmol}$ and $17.91 \mathrm{fmol}$ for both peptides in PSA per/ $50 \mu \mathrm{g}$. 22Rv1 cells are known to express PSA and are much more aggressive, which corresponds to our SID-SRM-MS results. Both $\mathrm{LnCaP}$ and $\mathrm{VCaP}$ cells are from highly aggressive PCA metastases, lymph node and bone, respectively. Both cell lines are known to express PSA. Peptide biomarkers for EZH2, AMACR, and both peptides in PSA were measured at 8.66, 22.67, and 89.89 and $176.00 \mathrm{fmol} /$ $50 \mu \mathrm{g}$ of total cellular protein, respectively, in LnCaP cells. While the same peptides measured 3.85, 16.76, 20.98, and $36.18 \mathrm{fmol}$, respectively per $50 \mu \mathrm{g}$ of total cellular protein in VCaP cells. These results are shown in Fig. 3. Shown below each line graph are Western blot analysis for each protein and a Glyceraldehyde 3-phosphate dehydrogenase (GAPDH) loading control, which correlate the SID-SRM-MS results, further providing confidence that these peptide biomarkers are indeed good representation of their respective proteins.

First, these cell line results demonstrate a proof of principle. This SID-SRM-MS methodology can accurately measure the presence or absence of PSA. As characterized by the American Type Tissue Culture (ATCC, Manassas, VA) the following cell lines do not express PSA: RWPE, 


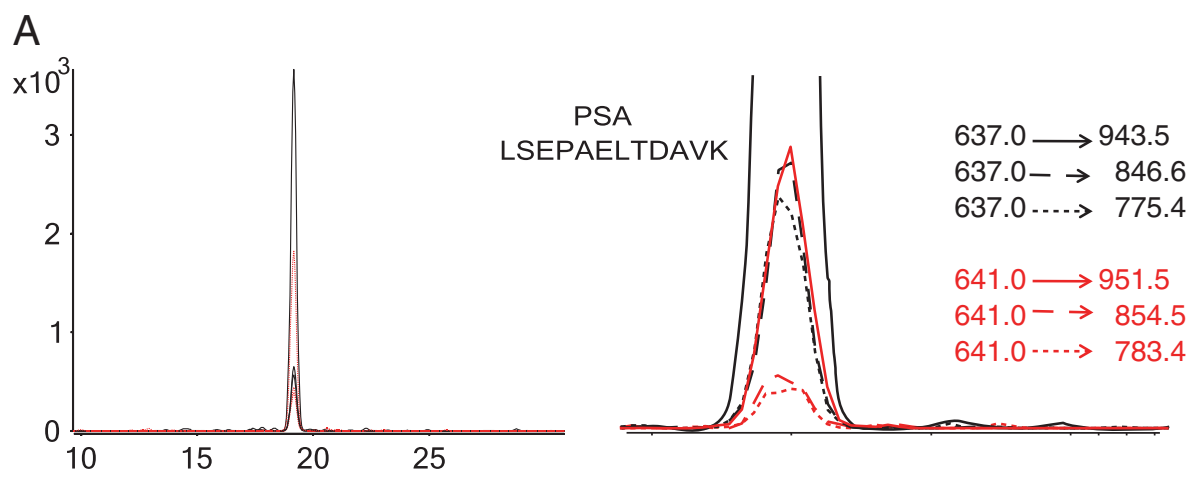

B

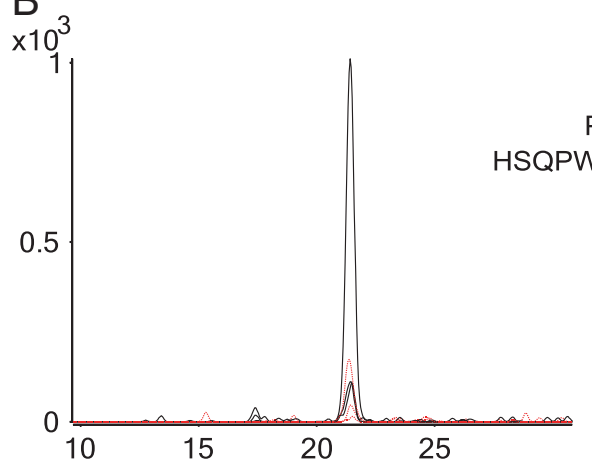

C
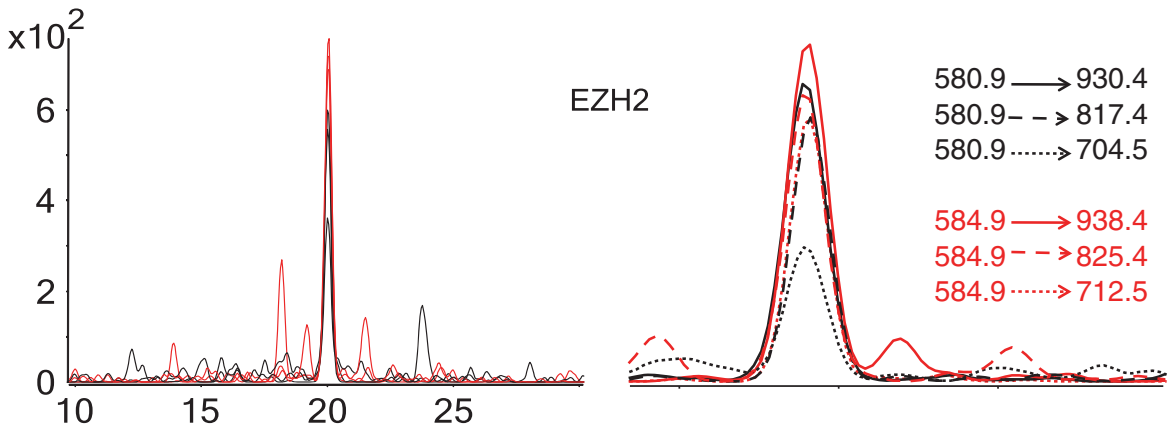

D

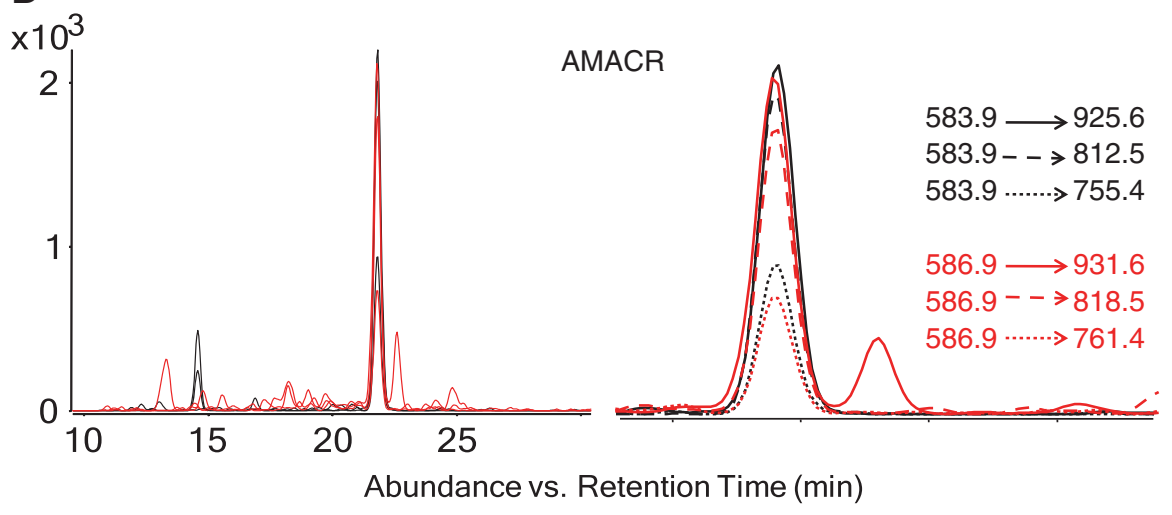

Figure 2. SID-SRM-MS traces of each peptide measured. EIC of spiked heavy peptide standard is outlined in red. EIC of light, i.e. endogenous peptide are in black. All six extracted transitions (three from heavy internal standard and three from endogenous) must overlap in retention time and be in relational agreement one another, as shown in the magnified version (left panel), to be considered a positive measurement. Representative EICs are taken from the most abundant fraction for each peptide. Both PSA peptides and the AMACR peptide were measured in LnCaP cells while EZH2 peptide was measured in 22Rv1 cells is shown.
PC3, and DU145 while the following do: 22Rv1, LnCaP, and VCaP. Our SID-SRM results emulate exactly that. Secondly, these results demonstrate that a panel of peptide biomarkers may provide a mechanism in which to tease out the intrinsic molecular properties specific to the peculiarities of PCA. For instance, in RWPE, PC3, and DU145 cells, both EZH2 and AMACR were measured at lower levels, suggesting a localized, non-aggressive phenotype. Opposing these results are 

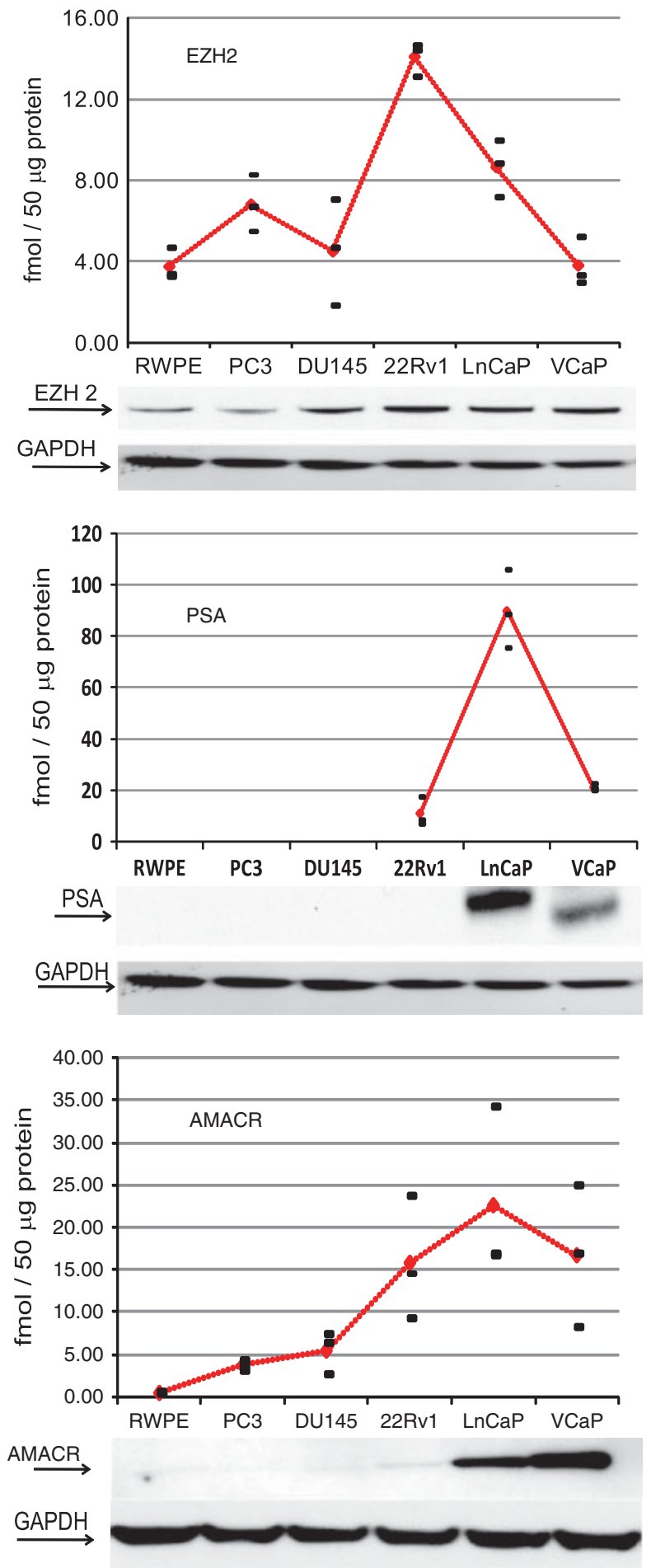

Figure 3. Proof of principle for SID-SRM-MS analysis with immortalized cells lines. Line graphs show the approximate concentrations for three biological replicates (black dashes) with an average and trend line (red diamonds and red dashed line, respectively) in six immortalized cell lines. Western blots for all three proteins are shown below respective line graphs with GAPDH as loading control.

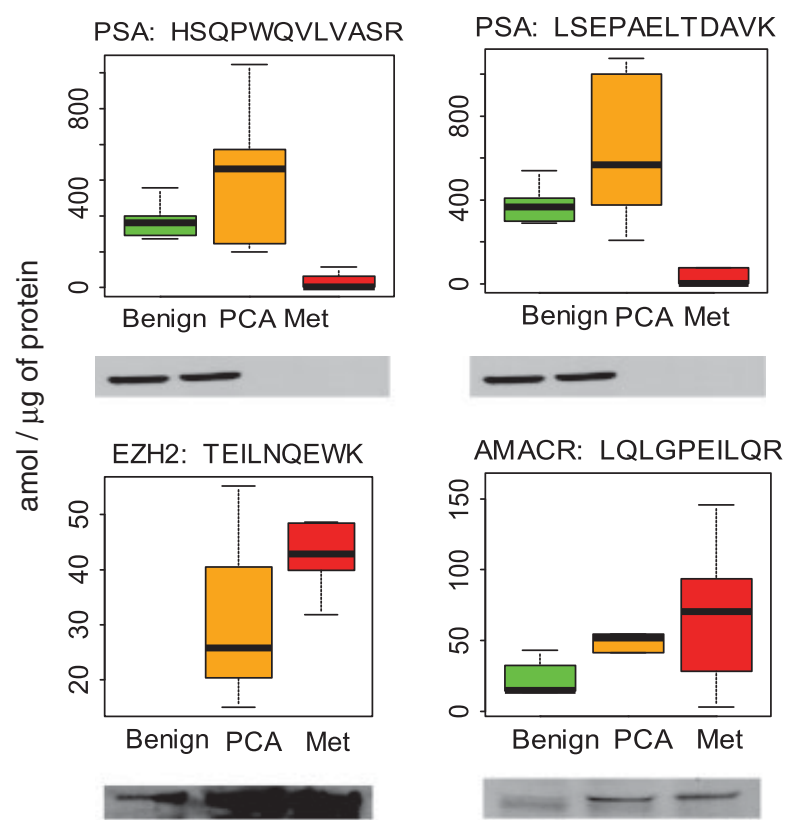

Figure 4. Measurement of peptide biomarkers in tissues. Box plots for the approximate concentration of each peptide biomarker measured in pooled tissue samples with SID-SRMMS. Western blots for each of the respective proteins are shown below box plots.

the higher measurements of AMACR in the aggressive, metastatic cell lines, 22Rv1, LnCaP, and VCaP. Interestingly, 22Rv1, an aggressive, hormone-independent cell line, showed very high levels of AMACR. This suggests that very high levels of AMACR may be indicative of hormone independence. Finally, it was surprising to see the low levels of EZH2 in the VCaP cell line. We speculate the following biological rationale for this finding. EZH2 is a histone methyl transferase that generally confers transcriptional repressional activity. It is hypothesized that the $\mathrm{VCaP}$ cell line contains so much gross genomic instability that the epigenetic transcription regulation is no longer a principle part of its cancerous properties.

\subsection{Tissues samples}

Next, the assay was transferred to clinical tissue samples, either prostate biopsy cores directly from histological positive localized tumor cells and adjacent benign tissue or samples taken from the location of major metastases at autopsy. A summary of results are shown in Fig. 4. Box plots show the range and average concentration of each peptide biomarker for each category of tissue samples. Below the box plots are Western blots for the target protein measured in pooled tissues samples for each category. As shown, the Western blots correlate the SID-SRM-MS measurements. Strikingly, the peptide biomarkers, EZH2 and AMACR, show an increased concentration, albeit 


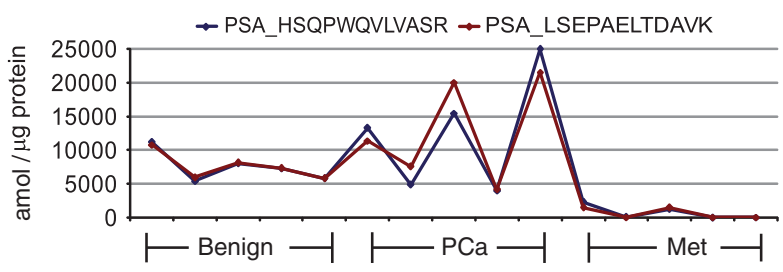

Figure 5. Two Peptides from the PSA protein correlate. Both peptides measured by SID-SRM-MS methodology correlate with one another across 15 different tissue samples.

non-statistically significant, in the metastatic tissue samples when compared with both benign adjacent and the localized tumor. Additionally, the concentration of EZH2, AMACR, and PSA show an increase, again not statistically significant, from benign adjacent to localized tumor. As expected, the concentration of PSA decreases $(p<0.01)$ with metastasis due to treatment initiation of these patients prior to death. It is believed that additional samples will increase the statistical significance of these preliminary findings.

Contained in the Supporting Information are the serum ELISA PSA results for the five metastatic samples measured. For purposes of correlating the two measurements, it is prudent to use the ELISA value at time of death as the tissue samples were taken at autopsy. It is, however, very difficult to directly compare the ELISA results with the SID-SRM-MS results due to the different locations of the sample matrix. It is expected that there is biotransformation and various protein kinetics adversely affecting a correlation of the different measurement simply because of diverse sample locations. This is in contrast to the excellent correlative work by Fortin et al. where both ELISA and MS measurements for PSA were taken in the serum $[15,18]$. While we believe these issues prevent direct comparison, the data does show a general correlation as the patients, M1 and M3, with the highest serum ELISA values, 928 and $3776 \mathrm{ng} /$ $\mathrm{mL}$, also show the highest PSA SID-MRM-MS results, 93 and $67 \mathrm{fmol} / 50 \mu \mathrm{g}$ of total protein, respectively. These PSA values, however, do not correlate with the total Gleason score. Patient M4 had a total Gleason score of 9 with a relatively low SID-MRM-MS $(0.25 \mathrm{fmol} / 50 \mu \mathrm{g}$ of total protein) and ELISA PSA value $(189 \mathrm{ng} / \mathrm{mL})$, while patient M3, with the highest ELISA and SID-MRM-MS PSA values, had a total Gleason score of 7. Furthermore, all normal and localized tumor samples have a total Gleason score of 7 , despite sometimes very high SID-MRM-MS results. This we feel further argues the need for more discriminatory means of diagnoses. Intriguingly, the two different peptide measurements for PSA are in agreement with one another. In Fig. 5, the measured concentrations of each peptide representing PSA are shown. This figure illustrates that while measurements were obtained for each individual peptide, one or the other could be used singly in the multiplexed assay as each peptide separately show roughly equivalent results.

\section{Concluding remarks}

This work describes the preliminary development of a peptide biomarker assay utilizing SID-SRM-MS for PCA. The assay concentrates on four peptide biomarkers that are representatives of known protein biomarkers of PCA. We believe these results are both novel with regard to the multiplexed detection of known biomakers of PCA and technologically advanced, representing a preliminary development of an SIDSRM-MS assay utilized on clinical samples. While preliminary assay development and subsequent results show promise, further study demonstrating the percent recovery during sample preparation and utilizing the assay on additional tissue samples will be required to demonstrate statistical significance and clinical feasibility. Future work is also ongoing, which includes the addition of several more peptide biomarkers and the technical transition to urine. More peptide biomarkers can be added, which will aid in the detailed delineation of clinical cases necessitating more personal tailored treatment options. Finally, we believe that a multiplexed SID-SRM-MS assay has the potential to achieve a higher discriminatory power for the detection of PCA than PSA alone or in combination with DRE.

The authors have declared no conflict of interest.

\section{References}

[1] Etzioni, R., Penson, D. F., Legler, J. M., di Tommaso, D. et al., Overdiagnosis due to prostate-specific antigen screening: lessons from U.S. prostate cancer incidence trends. J. Natl. Cancer Inst. 2002, 94, 981-990.

[2] Thompson, I. M., Pauler, D. K., Goodman, P. J., Tangen, C. M. et al., Prevalence of prostate cancer among men with a prostate-specific antigen level $<$ or $=4.0 \mathrm{ng}$ per milliliter. N. Engl. J. Med. 2004, 350, 2239-2246.

[3] Brawley, O. W., Ankerst, D. P., Thompson, I. M., Screening for prostate cancer. CA Cancer J. Clin. 2009, 59, 264-273.

[4] Taylor, B. S., Pal, M., Yu, J., Laxman, B. et al., Humoral response profiling reveals pathways to prostate cancer progression. Mol. Cell. Proteomics 2008, 7, 600-611.

[5] Keshishian, H., Addona, T., Burgess, M., Mani, D. R. et al., Quantification of cardiovascular biomarkers in patient plasma by targeted mass spectrometry and stable isotope dilution. Mol. Cell. Proteomics 2009, 8, 2339-2349.

[6] Kuhn, E., Addona, T., Keshishian, H., Burgess, M. et al., Developing multiplexed assays for troponin I and interleukin-33 in plasma by peptide immunoaffinity enrichment and targeted mass spectrometry. Clin. Chem. 2009, 55, 1108-1117.

[7] Addona, T. A., Abbatiello, S. E., Schilling, B., Skates, S. J. et al., Multi-site assessment of the precision and reproducibility of multiple reaction monitoring-based measurements of proteins in plasma. Nat. Biotechnol. 2009, 27, 633-641. 
[8] Anderson, N. L., Anderson, N. G., Pearson, T. W., Borchers, C. H. et al., A human proteome detection and quantitation project. Mol. Cell. Proteomics 2009, 8, 883-886.

[9] Duncan, M. W., Yergey, A. L., Patterson, S. D., Quantifying proteins by mass spectrometry: the selectivity of SRM is only part of the problem. Proteomics 2009, 9, 1124-1127.

[10] Cao, Q., Yu, J., Dhanasekaran, S. M., Kim, J. H. et al., Repression of E-cadherin by the polycomb group protein EZH2 in cancer. Oncogene 2008, 27, 7274-7284.

[11] Kuefer, R., Varambally, S., Zhou, M., Lucas, P. C. et al., alpha-Methylacyl-CoA racemase: expression levels of this novel cancer biomarker depend on tumor differentiation. Am. J. Pathol. 2002, 161, 841-848.

[12] Kumar-Sinha, C., Shah, R. B., Laxman, B., Tomlins, S. A. et al., Elevated alpha-methylacyl-CoA racemase enzymatic activity in prostate cancer. Am. J. Pathol. 2004, 164, 787-793.

[13] Takahara, K., Azuma, H., Sakamoto, T., Kiyama, S. et al., Conversion of prostate cancer from hormone independency to dependency due to AMACR inhibition: involvement of increased AR expression and decreased IGF1 expression. Anticancer Res. 2009, 29, 2497-2505.
[14] Varambally, S., Dhanasekaran, S. M., Zhou, M., Barrette, T. R. et al., The polycomb group protein EZH2 is involved in progression of prostate cancer. Nature 2002, 419, 624-629.

[15] Fortin, T., Salvador, A., Charrier, J. P., Lenz, C. et al., Clinical quantitation of prostate-specific antigen biomarker in the low nanogram/milliliter range by conventional bore liquid chromatography-tandem mass spectrometry (multiple reaction monitoring) coupling and correlation with ELISA tests. Mol. Cell. Proteomics 2009, 8, 1006-1015.

[16] Sherman, J., McKay, M. J., Ashman, K., Molloy, M. P., How specific is my SRM? The issue of precursor and product ion redundancy. Proteomics 2009, 9, 1120-1123.

[17] Sherman, J., McKay, M. J., Ashman, K., Molloy, M. P., Unique ion signature Mass Spectrometry: a deterministic method to assign peptide identity. Mol. Cell. Proteomics 2009, 8, 2051-2062.

[18] Fortin, T., Salvador, A., Charrier, J. P., Lenz, C. et al., Multiple reaction monitoring cubed for protein quantification at the low nanogram/milliliter level in nondepleted human serum. Anal. Chem. 2009, 81, 9343-9352. 